\title{
Role of serum androgens and prostate-specific antigen levels in males with androgenetic alopecia
}

\section{Original Article}

\author{
Hesham N. Khaled ${ }^{1}$,Azza M. Abdu Allah', Ahmad A. Abdelhameed ${ }^{1}$, Wafaa A. Shehata ${ }^{1}$
}

${ }^{1}$ Departments of Dermatology, Andrology and STDs, Faculty of Medicine, Menoufia University, ${ }^{2}$ Medical Biochemistry and Molecular Biology, Faculty of Medicine, Menoufia University, Menoufia, Egypt, and Faculty of Apllied Medical Sciences, Taibah University, Yanbou, KSA, ${ }^{3}$ Department of Dermatology and Andrology, Khanka Central Hospital, Qalyoubia, Egypt

\begin{abstract}
Background: Androgenetic alopecia (AGA) is the most common form of hair loss. It is caused by the continuous miniaturization of affected hair follicles. Genetic factors and androgenic factors especially dihydrotestosterone (DHT), which is a testosterone tissue metabolite, play major roles in the pathogenesis of AGA. Many studies suggest that AGA may be a marker of increased risk of prostate cancer.

Objective: The study aimed to examine the role of serum androgens and prostate-specific antigen (PSA) in males with AGA. Patients and Methods: The study included 80 participants: 40 men diagnosed with AGA and 40 age-matched healthy male volunteers as a control group. The authors also divided the patient group into two subgroups: frontal AGA group and vertical AGA group. All participants were subjected to measurement of serum level of testosterone, DHT, and PSA by enzyme-linked immunosorbent assay.

Results: The study revealed that the mean serum level of testosterone and DHT in patients with AGA was higher than that in the control group, and the mean serum level of testosterone in vertical AGA group was higher than that in frontal AGA group. There was no significant difference between patients and controls regarding the mean serum level of PSA.

Conclusion: This study showed that serum androgens play an important role in the development of AGA, and there is a strong association between serum level of androgens and staging of AGA, whether frontal or vertical.
\end{abstract}

Key Words: Androgens, androgenetic alopecia, prostate-specific antigen.

Received: 03 November 2019, Accepted: 13 January 2020

Corresponding Author: Hesham Nabil Khaled, Department of Dermatology, Andrology and STDs, Faculty of Medicine, Menoufia University, Menoufia, Egypt, Tel.: 00201005474528, E-mail: heshamnk@hotmail.com

ISSN: 2090-6048, September 2019 Vol. 9, No. 3

\section{INTRODUCTION}

Androgenetic alopecia (AGA) - also termed male-pattern alopecia, common baldness, and malepattern hair loss - is the most common type of alopecia occurring after puberty. It is typically manifested as progressive hair thinning and shortening in affected areas. Although regarded as a minor dermatological condition, it affects self-image and is a great cause of anxiety and depression in some patients, particularly younger ones $^{[1-3]}$. A link between male pattern baldness and androgens has previously been documented ${ }^{[4,5]}$. The effects of androgens on follicles vary depending on the body site. Although androgens stimulate beard growth and body hair in other sites in males, they suppress hair growth in the scalp in AGA. This reciprocal effect has been described as the androgen paradox ${ }^{[6]}$. Androgens also play a role in the development and growth of prostate cancer. That is why finasteride, which is a 5-areductase inhibitor that blocks the conversion of testosterone to dihydrotestosterone (DHT), is used for the treatment of AGA and has been shown to decrease the incidence of prostate cancer ${ }^{[7,8]}$. Despite this, a direct link between male pattern baldness and prostate cancer has not been consistently shown ${ }^{[9-14]}$. The authors have suggested that inconsistencies in results may be owing to differences in the patterns of hair loss and in the time frame of development of androgenic alopecia. AGA occurring in younger males (before the age of 30), as well as hair loss implicating the vertex, may be a precursor to developing prostate cancer later in life ${ }^{[6,12,15]}$.

\section{AIM OF THE WORK}

The aim was to study the role of serum androgens and prostate-specific antigen (PSA) on AGA by measuring their levels in serum of males with AGA and to correlate their levels with the available clinical data. 


\section{PATIENTS AND METHODS}

This case-control study was started after taking the approval of Dermatology and Andrology Department and Medical Ethics Committee, Faculty of Medicine, Menoufia University.

This case-control study was conducted on a total number of 80 participants: 40 male patients with AGA and 40 age-matched healthy male volunteers as a control group. The study included diagnosed males with AGA irrespective of age. It excluded patients with alopecia of other than androgenetic etiology (alopecia areata or cicatricial alopecia); patients who used finasteride or dutasteride, or any other drug capable of impairing androgen action (saw palmetto, antidepressants, GnRH agonists, isotretinoin, NSAIDs, statins, and thiazide)used in the last 6 months before the study initiation; patients with pre-existing medical conditions of the scalp that could affect the results of the study; and patients with benign prostatic hyperplasia, prostatic carcinoma diagnosis, urinary retention history, renal failure, urinary incontinence, urinary tract infection.

All studied patients were subjected to complete history taking, as well as clinical, general, and dermatologicalexaminations. Patients were classified according to the pattern of hair loss as per HamiltonNorwood classification system into 11 stages including stage I, II, III, III vertex, IIIa, IV, IVa, V, Va, VI, and VII. The patient group was also divided into two subgroups: frontal AGA group, which included stages I, II, III, IIIa, IV and IVa, and vertical AGA group, which included stages IIIvertex, V/Va/VI, and VII[16]. The blood sample was obtained from every participant under complete aseptic conditions by sterile venipuncture and put into a dry sterile plain tube for assessment of serum T, DHT, and PSA. Measurement of serum testosterone was doneby bio check testosterone enzyme immunoassay (EIA) kit manufactured by BioCheck Inc.(South San Francisco, California, USA). This kit is based on the principle of competitive binding between testosterone in the test specimen and testosteronehorseradish peroxidase conjugate, for a constant amount of rabbit antitestosterone. Measurement of serum DHT was determined by Human DHTenzyme-linked immunosorbent assay kit manufactured by Shanghai Sunred Biological Technology Co. This kit is based on the principle of the double-antibody sandwich technique. Measurement of PSA was determined by a human prostate-specific antigen enzyme-linked immunosorbent assay kit manufactured by Shanghai Sunred Biological Technology Co, Baoshan District, Shanghai, China. This kit is based on the principle of the double-antibody sandwich technique.

\section{Statistical analysis}

Data collected were tabulated and analyzed by SPSS (Statistical Package for the Social Science Software, Unicon Systems Inc. Los Angeles, California, USA) statistical package version 22 on IBM compatible computer. Quantitative data were expressed as mean and SD and analyzed by applying Student's t-test for comparison of two groups of normally distributed variables and Mann-Whitney U-test for non-normally distributed ones. Qualitative data were expressed as number and percentage and analyzed by applying a $\chi 2$-test.

\section{RESULTS}

The age of the patients ranged from 22 to 41 years, with mean \pm SD of $31.05 \pm 5.39$ years. Age of the controls ranged from 22 to 44 years, with mean $\pm \mathrm{SD}$ of $32.70 \pm 5.84$ years (Table 1).

Regarding other clinical data of the studied patients, the age of onset of AGA ranged from 18 to 30 years, with mean \pm SD of $23.15 \pm 2.88$ years. The course of AGA was progressive in $20(50 \%)$ cases and was stationary in 20 $(50 \%)$ cases. The family history of AGA was positive in $23(57.5 \%)$ cases (Table 1$)$.

Table 1: Clinical data of cases and controls $(\mathrm{No}=80)$

\begin{tabular}{|c|c|c|c|c|}
\hline \multirow{2}{*}{ Variables } & Cases & Controls & \multirow{2}{*}{ Test of Sig. } & \multirow{2}{*}{$P$. value } \\
\hline & $(\mathrm{No}=40)$ & $(\mathrm{No}=40)$ & & \\
\hline \multicolumn{5}{|l|}{ Age (years) } \\
\hline Range & $22-41$ & $22-44$ & \multirow{2}{*}{ T. test $=1.313$} & \multirow{2}{*}{$0.193 \mathrm{NS}$} \\
\hline Mean \pm SD & $31.05 \pm 5.39$ & $32.70 \pm 5.84$ & & \\
\hline \multicolumn{5}{|l|}{ Age of onset } \\
\hline Range & $18-30$ & & & \\
\hline Mean \pm SD & $23.15 \pm 2.88$ & & & \\
\hline
\end{tabular}




\begin{tabular}{llr}
\hline Course & & \\
Progressive & 20 & $50.0 \%$ \\
Stationary & 20 & $50.0 \%$ \\
Duration/year & \multicolumn{2}{c}{$1-24$} \\
Range & $9.53 \pm 4.99$ \\
Mean \pm SD & & \\
Family history & 23 & $57.5 \%$ \\
Positive & 17 & $42.5 \%$ \\
Negative & \\
\hline
\end{tabular}

Data were expressed as range, mean $\pm \mathrm{SD}$ and $\mathrm{n}(\%)$.

Student t-test was used for comparison between quantitative variables of the study groups. Cases and controls.

$P$ value less than 0.05 was considered statistically significant

According to Norwood Hamilton classification system, five $(12.5 \%)$ cases were stage I, five $(12.5 \%)$ cases were stage II,three $(7.5 \%)$ cases were stage III, $14(35 \%)$ cases were stage III vertex,two (5\%) cases were stage IIIa,one $(2.5 \%)$ case was stage IV,four $(10 \%)$ cases were stage Iva,one $(2.5 \%)$ case was stage $\mathrm{V}$, three $(7.5 \%)$ cases were stage $\mathrm{Va}$, one $(2.5 \%)$ casewas stage VI, one $(2.5 \%)$ case was stage VII (Table 3). There were 20 (50\%) cases classified as frontal AGA including stages I, II, III, IIIa, IV, and IVa, and $20(50 \%)$ cases were classified as vertical AGA, including stages III vertex, V, Va, VI, and VII (Table 2).

There was a negative correlation $(\mathrm{r}= \pm 0.282)$ between mean serum level of testosterone and duration of AGA in the studied patients (Table 3 ). The mean serum level (mean $\pm \mathrm{SD}$ ) of $\mathrm{T}$ in cases was $9.46 \pm 3.78$, $\mathrm{ng} / \mathrm{dl}$ and in controls was $2.58 \pm 1.39$ ng/dl, with Pvalue less than 0.001 (Table 4, Fig.1).

The mean serum level (mean \pm SD) of DHT in cases was $604.30 \pm 1093.07 \mathrm{pg} / \mathrm{ml}$ and in controls was $37.87 \pm 15.45 \mathrm{pg} / \mathrm{ml}$, with $P$ value of 0.002 (Table 4).

The mean serum level (mean $\pm \mathrm{SD}$ ) of PSA in cases was $0.46 \pm 0.13 \mathrm{ng} / \mathrm{ml}$ and in controls was $0.43 \pm 0.07$ ng/ml, with $P$ value greater than 0.05 (Table 4, Fig.1).

Table 2: Staging of studied cases according to Norwood Hamilton grading system $(n=40)$

\begin{tabular}{|c|c|c|c|}
\hline Stages & Frontal $(\mathrm{n}=20)[\mathrm{n}(\%)]$ & Vertical $(\mathrm{n}=20)[\mathrm{n}(\%)]$ & Total $(\mathrm{n}=40)[\mathrm{n}(\%)]$ \\
\hline I & - & - & $5(12.5)$ \\
\hline II & - & - & $5(12.5)$ \\
\hline III & - & - & $3(7.5)$ \\
\hline III vertex & $14(70)$ & $14(70)$ & $14(35.0)$ \\
\hline IIIa & - & - & $2(5.0)$ \\
\hline IV & - & - & $1(2.5)$ \\
\hline Iva & - & - & $4(10.0)$ \\
\hline $\mathrm{V}$ & $1(5)$ & $1(5)$ & $1(2.5)$ \\
\hline $\mathrm{Va}$ & $3(15)$ & $3(15)$ & $3(7.5)$ \\
\hline VI & $1(5)$ & $1(5)$ & $1(2.5)$ \\
\hline VII & $1(5)$ & $1(5)$ & $1(2.5)$ \\
\hline Total & $20(50)$ & $20(50)$ & $40(100)$ \\
\hline
\end{tabular}

Staging of androgenetic alopecia in studied cases according to Norwood Hamilton grading system was expressed asn(\%). 
Table 3: Correlation between mean serum level of testosterone, dihydrotestosterone, and prostate-specific antigen and duration of androgenetic alopecia of the studied cases.

\begin{tabular}{lcc}
\hline & & Duration \\
Hormones & $\mathrm{r}$ & $P$ value \\
\cline { 2 - 3 } $\mathrm{T}$ & -0.282 & $0.078(\mathrm{NS})$ \\
DHT & 0.029 & $0.861(\mathrm{NS})$ \\
PSA & 0.105 & $0.520(\mathrm{NS})$ \\
\hline
\end{tabular}

Correlation(r) was used to express the relation between mean serum level of hormones T, DHT, PSA and duration of AGA in studied cases. DHT, dihydrotestosterone; PSA, prostate-specific antigen; T, testosterone.

$P$ value less than 0.05 was considered statistically significant.

Table 4: Comparison between cases and controls regarding mean serum levels of testosterone, dihydrotestosterone, and prostate-specific antigen

\begin{tabular}{|c|c|c|c|c|}
\hline Hormones & Cases $(n=40)$ & Controls $(n=40)$ & t-test & $P$ value \\
\hline \multicolumn{5}{|l|}{$\mathrm{T}$} \\
\hline \multirow[t]{2}{*}{ Range } & $2.9-17.7$ & $0.26-5.95$ & \multirow{3}{*}{10.786} & 0.001 \\
\hline & & & & HS \\
\hline Mean \pm SD & $9.46 \pm 3.78$ & $2.58 \pm 1.39$ & & \\
\hline \multicolumn{5}{|l|}{ DHT } \\
\hline \multirow[t]{2}{*}{ Range } & $30.65-6785.7$ & $11.81-71.74$ & \multirow{3}{*}{3.277} & \multirow{3}{*}{$\begin{array}{c}0.002 \\
\text { HS }\end{array}$} \\
\hline & & & & \\
\hline Mean \pm SD & $604.30 \pm 1093.07$ & $37.87 \pm 15.45$ & & \\
\hline \multicolumn{5}{|l|}{ PSA } \\
\hline \multirow[t]{2}{*}{ Range } & $0.16-1.06$ & $0-0.5$ & \multirow{3}{*}{1.099} & \multirow{3}{*}{$\begin{array}{c}0.275 \\
\text { NS }\end{array}$} \\
\hline & & & & \\
\hline Mean \pm SD & $0.46 \pm 0.13$ & $0.43 \pm 0.07$ & & \\
\hline
\end{tabular}

Data were expressed as mean $\pm \mathrm{SD}$ and range.

Student t-test was used for comparison between mean serum levels of hormones; T, DHT, and PSA of the study groups. Cases and controls. DHT, dihydrotestosterone; HS, highly significant; PSA, prostate-specific antigen; T, testosterone.

$P$ value less than 0.05 was considered statistically significant. 


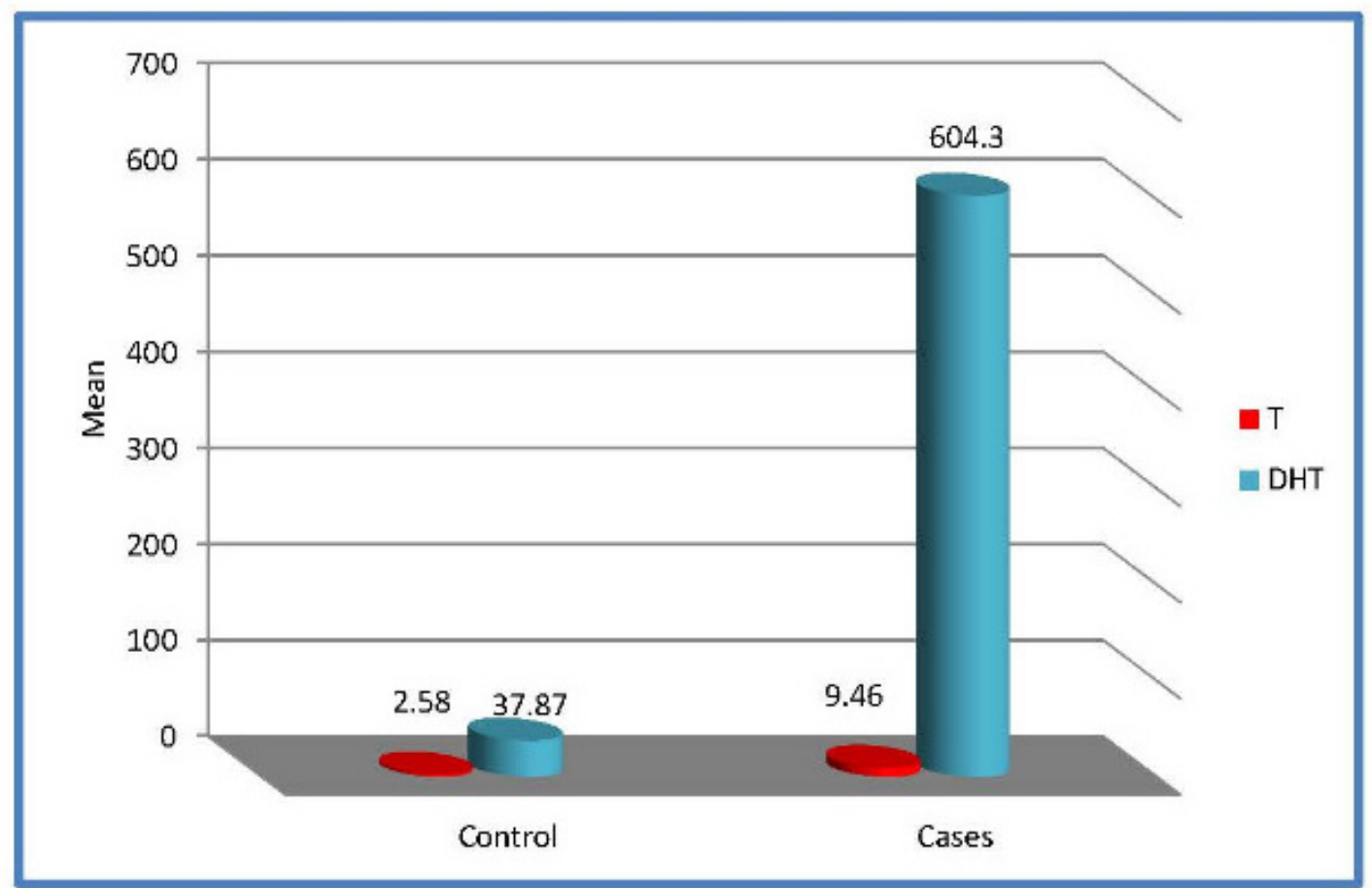

Fig. 1: Comparison between cases and controls regarding mean serum level of testosterone and dihydrotestosterone $(\mathrm{n}=80)$.

Table 5: Comparison between cases with frontal androgenetic alopecia and cases with vertical androgenetic alopecia regarding mean serum level of testosterone, dihydrotestosterone, and prostatic specific antigen

\begin{tabular}{|c|c|c|c|c|}
\hline & \multicolumn{2}{|c|}{ Staging } & \multirow[b]{2}{*}{ t-test } & \multirow[b]{2}{*}{$P$ value } \\
\hline & Frontal & Vertical & & \\
\hline \multicolumn{5}{|l|}{ Hormones } \\
\hline Range & $4.52-13.38$ & $5.41-13.4$ & \multirow{2}{*}{4.3} & \multirow{2}{*}{$\begin{array}{c}0.001 \\
\text { HS }\end{array}$} \\
\hline Mean \pm SD & $7.89 \pm 2.6$ & $12.36 \pm 3.99$ & & \\
\hline \multicolumn{5}{|l|}{ DHT } \\
\hline Range & $68.97-260.44$ & $30.65-6785.7$ & \multirow{2}{*}{0.29} & \multirow{2}{*}{$\begin{array}{l}0.78 \\
\text { NS }\end{array}$} \\
\hline Mean \pm SD & $567.64 \pm 1348.8$ & $672.38 \pm 279.22$ & & \\
\hline \multicolumn{5}{|l|}{ PSA } \\
\hline Range & $0.41-0.52$ & $0.43-0.46$ & \multirow{2}{*}{0.305} & \multirow{2}{*}{$\begin{array}{c}0.76 \\
\text { NS }\end{array}$} \\
\hline Mean \pm SD & $0.46 \pm 0.079$ & $0.45 \pm 0.2$ & & \\
\hline
\end{tabular}

-Data were expressed as mean $\pm \mathrm{SD}$ and range.

-Student t-test was used for comparison between mean serum levels of hormones; T, DHT, and PSA in cases with frontal AGA and cases with vertical AGA.

-AGA, androgenetic alopecia; DHT, dihydrotestosterone; HS, highly significant; PSA, prostate-specific antigen; T, testosterone.

$P$ value less than 0.05 was considered statistically significant. 


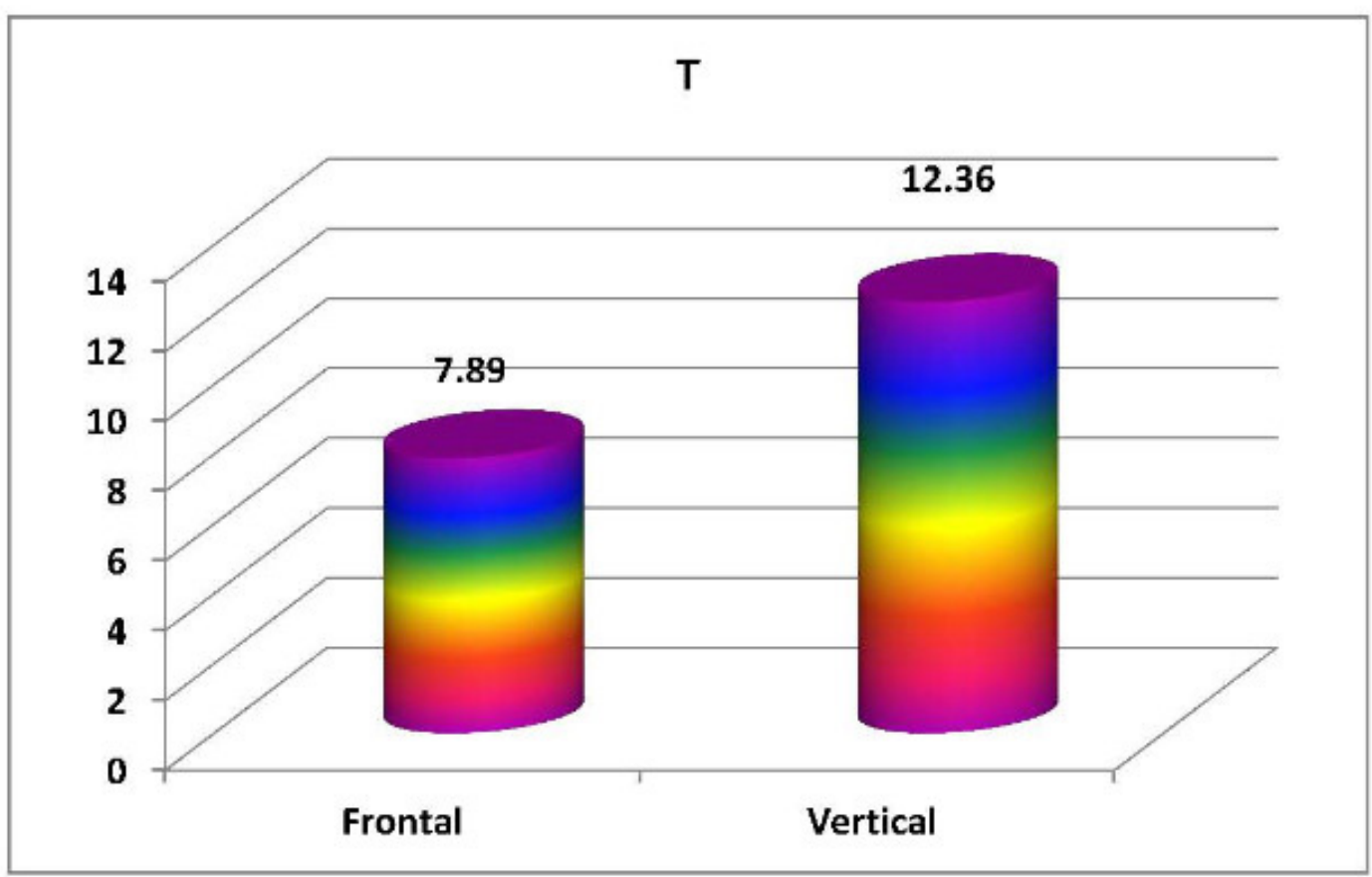

Fig. 2: Comparison between cases with frontal androgenetic alopecia and cases with vertical androgenetic alopecia regarding mean serum level of testosterone.

\section{DISCUSSION}

In our study, of 40 patients with AGA, 23(57.5\%) cases had a positive family history of AGA, which supports the genetic predisposition in AGA. This result was nearly similar to a study by Devi et al. ${ }^{[17]}$, who conducted a study on 27 males clinically diagnosed as having AGA and reported that $66.7 \%$ of the patients had a positive family history of AGA.

In this study, a negative correlation was found between mean serum level of testosterone and duration of AGA, denoting that there is a steady progressive deterioration of serum $\mathrm{T}$ level with age, which is in agreement with a study by Lapauw et al. ${ }^{[18]}$, who found a negative correlation between mean serum level of testosterone and aging in men regardless having AGA or not.

Our study showed that there was a statistically significant difference between cases and controls regarding the mean serum level of testosterone $(P<0.001)$ being higher in AGA cases. This result was in agreement with a study by Narad et $a l .{ }^{[19]}$, and a study by Sanke et $a l .{ }^{[20]}$.

In this study, there was a statistically significant difference between cases and controls regarding the mean serum level of DHT ( $P=0.002)$, being higher in cases than in controls. This result was in agreement with a study by Bang et al. ${ }^{[21]}$, and a study by Zhang et al. ${ }^{[22]}$. However, no significant difference between cases and controls regarding the mean serum level of PSA was found.

In this study, there was a statistically significant difference between vertical AGA group and frontal AGA group regarding mean serum level of testosterone $(P<0.001)$, being higher in vertical AGA than in frontal AGA group, but there was no significant difference between frontal AGA group and vertical AGA group regarding mean serum level of DHT or PSA. Such finding has no clear explanation in the literature, but we think that it may be because frontal hair follicles are more sensitive to androgen action, and it starts the process of miniaturization even at low levels of androgen in comparison with vertical hair follicles, whichneed higher levels of androgen.

In the present study, no clear connection was found between serum levels of T, DHT, or PSA and clinical grades of AGA in patients, denoting that an advanced case of AGA does not necessarily mean a higher level of androgens in comparison with early cases of AGA and vice versa.

However, we believe that the relationship between AGA and androgens and also the exact effectof androgens on the etiopathogenesis of AGA need further studies on larger groups of patients. Moreover,whether AGA in the male can point to the possibility of having prostate cancer in the future is a point of debate,which may be clarified shortly by more deep studies on the possible link between AGA and prostate cancer. 


\section{CONCLUSION}

From this work, we could conclude the following:

(1) Patients with AGA have higher serum levels of testosterone and DHT than normal healthy participants.

(2) Serum level of androgens plays an important and crucial role in the development of AGA.

(3) There is a strong association between serum level of androgens and the staging of AGA whether frontal or vertical.

(4) Genetic predisposition plays an important role in the development of AGA.

(5) There is no association between AGA and the risk of development of prostate cancer.

\section{RECOMMENDATION}

The intricate relationship between androgens and AGA necessitates further studies on larger groups of patients. This may ultimately help in finding an effective treatment for AGA that targets reducing androgen levels only on the level of the scalp without affecting serum levels of androgens.

\section{CONFLICT OF INTEREST}

There are no conflicts of interest.

\section{REFERENCES}

1. PiracciniBM, AlessandriniA. Androgenetic alopecia. G Ital Dermatol Venereol2014; 149; 15-24.

2. DeMuro-MerconC, RhodesT, GirmanCJ, VattenL. Male-pattern hair loss in Norwegian men: a community-based study. Dermatology 2000; 200:219-222.

3. LeeWS, LeeHJ. Characteristics of androgenetic alopecia in Asian. Ann Dermatol2012; 24:243-252.

4. Vierhapper H, Nowotny $\mathrm{P}$, Maier $\mathrm{H}$, WaldhauslWProduction rates of dihydrotestosterone in healthy men and women and men with male pattern baldness: determination by stable isotope/dilution and mass spectrometry. $\mathrm{J}$ Clin Endocrinol Metab 2001; 86:5762-5764.

5. Ellis JA, HarrapSBThe genetics of androgenic alopecia. Clin Dermatol2001; 19: 149-154.

6. InuiS, ItamiS. Androgen actions on the human hair follicle: perspectives. Exp Dermatol 2013; 22:168-171.

7. ThompsonIM, GoodmanPJ, TangenCM, LuciaMS, MillerGJ, FordLG, et al. The influence of finasteride on the development of prostate cancer. N Engl JMed2003; 349:215-224.

8. VickersAJ, SavageCJ, LiljaH. Finasteride to prevent prostate cancer: should all men or only a high-risk subgroup be treated?. J Clin Oncol 2010; 28:1112-1116.

9. Demark-WahnerfriedWE, LeskoSM, ConawayMR, RobertsonCN, ClarkRV, LobaughB, et al. Serum androgens: associations with prostate cancer risk and hair patterning. J Androl1997; 18:495-500.

10. HsiehCC, ThanosA, MitropoulosD, DeliveliotisC, MantzorosCS, TrichopoulosD. Risk factors for prostate cancer: a case-control study in Greece. Int J Cancer1999; 80:699-703.

11. Demark-WahnefriedW, SchildkrautJM, ThompsonD, LeskoSM, McIntyreL, SchwinglP, et al. Early-onset baldness and prostate cancer risk. Cancer Epidemiol Prev Biomarkers2000; 9:325-328.

12. HawkE, BreslowRA, GraubardBI. Male pattern baldness and clinical prostate cancer in the epidemiologic follow-up of the first National Health and Nutrition Examination Survey. Cancer Epidemiol Biomarkers Prev 2000; 9:523-527.

13. GilesGG, SeveriG, SinclairR, EnglishDR, McCredieMR, JohnsonW, et al. Androgenetic alopecia and prostate cancer: findings from an Australian case-control study. Cancer Epidemiol Prev Biomarkers2002; 11:549-553.

14. FaydaciG, BilalE, NecmettinP, FatihT, AsumanO, UğurK. Baldness, benign prostate hyperplasia, prostate cancer, and androgen levels. Aging Male2008; 11:189-192.

15. Demark-WahnefriedW, SchildkrautJM. Male pattern baldness and clinical prostate cancer in the epidemiologic follow-up of the First National Health and Nutrition Examination Survey. Cancer Epidemiol Prev Biomarkers 2001; 10:415-416.

16. NorwoodOT. Male pattern baldness: classification and incidence. South Med J1975; 68:1359-1365.

17. DeviN, ChintaguntaSR, ArakkalG, NagulaSK. Androgenetic alopecia- Role of androgen levels. Int J Biomed Res2016; 7:410-413. 
18. LapauwB, GoemaereS, ZmierczakH, Van PottelberghI, MahmoudA, TaesY, et al. The decline of serum testosterone levels in communitydwelling men over 70 years of age: descriptive data and predictors of longitudinal changes. Eur J Endocrinol2008; 159:459-468.

19. NaradS, PandeS, GuptaM, ChariS. Hormonal profile in Indian men with premature androgenetic alopecia. Int J Trichol2013; 5:69-72.

20. SankeS, ChanderR, GargT, JainA. Free Androgen Index (FAI): marker of premature androgenetic alopecia in men. J Microb Biochem Technol 2016; 8:097-099.

21. BangHJ, YangYJ, LhoDS, LeeWY, SimWY, ChungBC. Comparative studies on level of androgens in hair and plasma with premature malepattern baldness. J Dermatol Sci2004; 34:11-16.

22. ZhangY, XuJ, Jing, J, WuX, LvZ. Serum levels of androgen-associated hormones are correlated with curative effect in androgenic alopecia in young men. Med Sci Monitor 2018; 24:7770-7777. 